\title{
Vesicovaginal fistula: what is the preferred closure technique?
}

\author{
Edward Stanford • Lauri Romanzi
}

Received: 7 October 2011 / Accepted: 16 November 2011 /Published online: 8 December 2011

(C) The International Urogynecological Association 2011

Vesicovaginal fistula (VVF) is a worldwide problem; however, the true incidence and prevalence is not known. Reportedly affecting as many as two million women, this number reflects an estimate from an expert opinion, without the benefit of national or regional "interview and exam" prevalence data [1]. In most settings where obstetric fistula presents an ongoing public health problem, fistula incidence is extrapolated from maternal mortality estimates (as high as 1:7 in impoverished settings compared to 1:4,000 in industrialized nations) if calculated at all. Accurate global prevalence data are difficult to collect, particularly in global regions with poor infrastructure and large populations living in remote rural settings where obstetric fistula is most common.

In the past 150 years, we have seen that VVF from obstetrical injury can be and has been virtually eliminated when timely obstetrical care is provided. In more developed countries, there has been a dramatic shift and VVF are most often reported after major gynecologic surgery (usually hysterectomy [2]), pelvic malignancy, or radiation treatment. Yet, even in these industrialized nations, the genital fistula epidemiology is incomplete and the actual prevalence of VVF resulting from gynecologic surgery remains poorly documented.

It is quite interesting to review briefly the history of vaginal VVF surgery which dates back to Pharaonic parchments. Unlike many gynecologic surgeries which have a

\footnotetext{
E. Stanford $(\bowtie)$

Urogynecology Private Practice,

Chicago, IL, USA

e-mail: ejs222@aol.com

L. Romanzi

Weill Cornell Medical Center/New York Presbyterian Hospital, New York, NY, USA
}

shorter history, modern vaginal VVF surgery date back to surgical attempts around 1663 with the first reported successful closure by Johann Fatio in 1675. Vaginal skin flaps and tension-free closure were adopted in the $1830 \mathrm{~s}$ predating the landmark work by J. Marion Sims in 1852 on a slave named Anarcha who after 30 attempts using silver wire achieved successful vaginal closure [3]. Further study of the history of VVF surgery reveals many historically interesting modifications in technique: Collis 1861-multilayered closure; Schuchardt 1893-pararectal incision to increase exposure; Maisonneuve mid-1880s - separate bladder from vagina; Kelly 1896-vaginal VVF and 1906 abdominal approach; Garlock 1928 - gracilis flap and ureteral catheters; and Martius 1928-bulbocavernosus flap. In 1942, Latzko introduced a vaginal technique for small accessible VVF [4] which many consider the standard vaginal repair technique.

Although, the original Hippocratic oath relegated the (abdominal) removal of vesical calculi to trained practitioners (lithotomists), it was not until around the mid 1880s that Trendelenburg introduced the abdominal suprapubic approach for VVF. Further, it was not until around 1950 that the O'Conor abdominal repair for the supratrigonal VVF [5] was introduced in which the bladder is bivalved. This technique has been considered the current gold standard abdominal approach.

Worldwide, the most common VVF is secondary to obstructed labor in countries in which obstetrical care is not readily available. The preferred route of the vast majority of fistula surgeons in those settings is a vaginal approach usually under regional anesthesia consistent with the resources available. Even though success is dictated by the complexity of the fistula or fistulas, it is quite amazing how successful the surgeons in these low-resource settings are overall. Another interesting observation is that, in some 
countries where obstetrical fistulae are common, the fistula surgeons may elect to repair obstetrical fistula vaginally if the injury is secondary to vaginal pressure necrosis. However, if the fistula is related to an abdominal delivery, the surgeon may elect to repair the fistula abdominally although there is no clear evidence-based support in the medical literature. Given the great variability of conditions from region to region and country to country, regional political stability, and whether the fistula is gynecologic or obstetric in origin, the most effective approach to repair VVF remains within the confines the surgeon's experience and technical skills.

The pace of fistula repair innovation has slowed to a near standstill since O'Conor's abdominal bivalve technique with some recent works regarding fulguration with cautery and laser treatment for carefully selected very small genital fistulae $[6,7]$. In the past decade, gynecologic VVF repair methods using advanced abdominal approaches via laparoscopic techniques (traditional and robotic) [8] have gained popularity in developed nations, an option neither practical nor accessible in the poorest nations plagued with the highest rates of obstetric fistulae and restricted resources.

Regardless of fistula etiology or the surgical approach used, the basic principles of VVF repair apply. Couvelaire put it simply in 1953 - good visualization, good dissection, good approximation of the margins, and good urinary drainage [9]. More succinctly, these principles include the absence of induration and excessive inflammation, a tension-free bladder closure in single or multiple layers, preservation of adequate vascular supply, separate layer closure of the vagina, avoidance of overlapping suture lines, and continuous bladder drainage postoperatively. In some cases a urinary or colorectal diversion may be required, raising the specter of lifelong diversion and ostomy management, to be carried out with the highest degree of planning, education, forethought, and caution in settings of poor infrastructure and high illiteracy. Complications of diversion include urinary tract infection, hyperchloremic metabolic acidosis, anal incontinence, occasionally adenocarcinoma, and death [10].

All experienced fistula surgeons agree that the first repair offers the greatest possibility of success. They also agree that no fistula, even those deemed "simple", should be repaired by inexperienced surgeons or in poor surgical theater conditions. A flexible utilization of approaches, including double setup for vaginal closure with possible conversion to abdominal repair, may be the optimal situation for closure at any initial fistula repair surgery. Most fistula surgeons agree that fistula tract excision, partial colpocleisis (Latzko), J-shaped incision, and the flap-splitting technique suffice individually or in combination for vaginal approach repairs, the success of which correlate to fistula classification and primary versus repeat repairs.
As with all reconstructive operations, the selection of VVF repair method is influenced by the surgeon's experience. Surgeons in low-resource areas where most fistulae are obstetrical may develop expertise weighted toward vaginal repairs. By contrast, surgeons exposed primarily to gynecologic fistulae may be more comfortable with abdominal or laparoscopic repairs involving variations of O'Conor's technique. This is particularly true in settings where ureteral injury after hysterectomy is more common. Another factor in choosing a repair is surgical volume. A surgeon will not become expert if exposed only to the occasional fistula. Ideally, the experienced VVF surgeon would be skilled in vaginal and abdominal techniques and have a relatively high volume of exposure, allowing for optimal informed consent and a flexible, tailored approach suitable to the specific VVF architecture and level of complexity [11].

Surgeons must embrace the patient's perspective. We now understand that "closing the hole" may not suffice in restoring quality of life and return to family and community for women with vaginal fistula involving the urethra (continence mechanism) or for VVF repairs resulting in ultra-low capacity, poor compliance bladders, both typically resulting in severe post-fistula repair urinary incontinence even when the fistula itself is successfully closed. The most common genital fistula etiology in countries lacking ready access to emergency obstetrical care is obstructed labor, often lasting days, swelling the ranks of maternal mortality and leaving a trail of clinical morbidities among the survivors, including vaginal fistulae, foot drop, vaginal stenosis-fibrosis, cloacal defects, and infertility. Regardless of etiology and method of repair, the patient's goals, including restoration of vaginal anatomy sufficient for coitus, successful closure of the fistula, and continence of urine and stool, remain the journey shared by fistula sufferer and fistula surgeon.

The proverbs "there are many ways to skin a cat" and "when all you have is a hammer, everything looks like a nail" both apply here. As medicine in underdeveloped areas advances, surgeons will adopt contemporary technologies used in the health care systems of wealthy nations. Surgeons from high-prevalence obstetric fistula areas will continue to expand their repertoires. It is understandable that for surgeons with more exposure to postoperative gynecologic fistula, their expertise may skew toward abdominal repairs including laparoscopic methods in technology-rich environments. We reiterate however that for most VVF, the preferred approach is vaginal. Abdominal repairs are reserved for cases involving difficult vaginal access, high VVF, ureteral involvement necessitating reimplantation, or the need for a concomitant abdominal procedure. 


\section{References}

1. WHO (2010) 10 facts on obstetric fistula. Available at http://www. who.int/features/factfiles/obstetric fistula/en/

2. Lee RA, Symmonds RE, Williams TJ (1988) Current status of genitourinary fistula. Obstet Gynecol 72:313-319

3. Spettel S, White MD (2011) The portrayal of J. Marion Sims' controversial surgical legacy. J Urol 185(6):2424-2427

4. Latzko W (1942) Postoperative vesicovaginal fistulas. Am J Surg 58:211

5. O'Conor VJ Jr (1980) Review of experience with vesicovaginal fistula repair. J Urol 123(3):367-369

6. Shah SJ (2010) Role of day care vesicovaginal fistula fulguration in small vesicovaginal fistula. J Endourol 21(10):1659-1660
7. Dogra PN, Saini AK (2011) Laser welding of vesicovaginal fistula-outcome analysis and long-term outcome: single centre experience. Int Urogynecol J 22(8):981-984

8. Wong C, Lam PN, Lucente VR (2006) Laparoscopic transabdominal transvesical vesicovaginal fistula repair. J Endourol 20(4):240-243

9. Couvelaire R (1953) Reflections on a personal statistic of 136 vesicovaginal fistulas. J Urol Medicale Chir 59:150 160

10. Azimuddin K, Khubchandani IT, Stasik JJ, Rosen L, Reither RD (1999) Neoplasia after ureterosigmoidostomy. Dis Colon Rectum 42(12):1632-1638

11. Blaivas JF, Heritz DM, Romanzi LJ (1995) Early versus late repair of vesicovaginal fistulas: vaginal and abdominal approaches. J Urol 153(4):1110-1112 